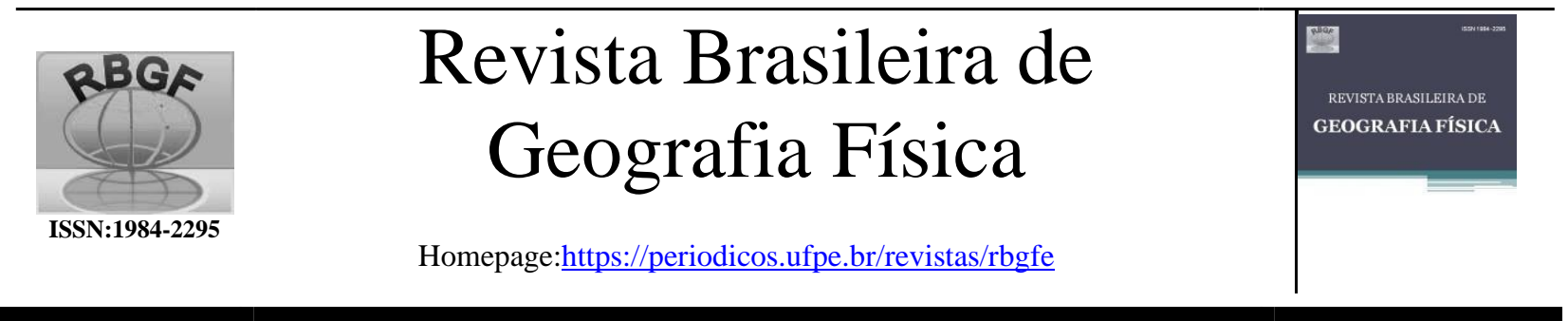

\title{
Mudanças espaço-temporais no uso e cobertura da terra na bacia hidrográfica do rio Pontal a partir de dados referenciais do Google Earth Pro
}

\author{
Viviane Pedroso Gomes ${ }^{1}$, Maria do Socorro bezerra de Araújo², Josiclêda Domiciano Galvíncio ${ }^{3}$
}

\begin{abstract}
1Doutoranda em Desenvolvimento e Meio Ambiente - PRODEMA, Universidade Federal de Pernambuco - UFPE. Autor correspondente: E-mail: vivianegomesgeo@gmail.com. ${ }^{2}$ Docente, Universidade Federal de Pernambuco. Departamento de Ciências Geográficas. Programa de Pós-graduação em Desenvolvimento e Meio Ambiente - PRODEMA, E-mail: socorro@ufpe.br. ${ }^{3}$ Docente, Universidade Federal de Pernambuco. Departa mento de Ciências Geográficas. Programa de Pós graduação em Desenvolvimento e Meio Ambiente - PRODEMA, E-mail: josicleda.galvincio@ufpe.br.
\end{abstract}

Artigo recebido em 13/07/2021 e aceito em 01/12/2021

\section{R E S U M O}

O uso e cobertura da terra compreende as diferentes estrutura modela das a partir de forças de ordens naturale antropogênica. Esse tem sido um dos a tributos mais utilizados para monitoramento e planejamento a mbiental. O objetivo do presente estudo foi ana lisar a mudanças espaço-temporais do uso e cobertura da terra na ba cia hidrográfica do rio Pontal, localizada no estado de Perna mbuco. Para isso, foram empregadas técnicas de geoprocessa mento e sensoria mento remoto utilizando cla ssificação supervisionada em imagens orbitais dos satélites Landsat 5 e 8. Para obtenção de pontos de controle optou-se por utilizar as informações do Google Earth Pro, que possui um banco com imagens de alta resolução espacial e assim possibilita o reconhecimento real da área. Os resultados encontrados indicaram, de modo geral, que a metodologia se mostrou viável no sentido de detectar a s classes de uso e cobertura da terra encontradas a longo da área de estudo. A bacia do Pontal possui hoje menos água disponível no seu balanço hídrico para os diferentes usos e sustentabilidade ambiental devido as mudanças climáticas globais e mudanças no uso e ocupação do solo. As técnicas empregadas para o mapeamento do uso e cobertura da bacia hidrográfica do rio Pontal se mostraram consistentes no sentido de representar a variação espacial e temporal da vegetação na referida unidade.

Pala vras-chave: Impacto a mbiental; classificação supervisiona da; Sensoria mento remoto.

\section{Space-temporal changes in land use and coverage in the Pontal river basin based on reference data from Google Earth Pro}

\begin{abstract}
A B S T R A C T
Land use and land cover are structures modeled afternaturaland anthropogenic orders. This has been one of the most used attributes for environmental monitoring and planning. The objective of the present study was analyzed as spatio-temporal changes in land use and land cover in the Pontal river basin, located in the state of Pernambuco. For this, geoprocessing and remote sensing techniques were used using supervised classification in images from Landsat 5 and 8 satellites. spatial resolution, thus enabling real recognition of the area. The results found indicate, in general, that one methodology showed no sense of detection as viable use classes and land cover along the study area. The Pontal basin today has water available in its water balance for different environmentaluses and environmental sustainability and changes in use and less land occupation. The techniques used for the use mapping and tip covera ge mentioned.

Keywords: Environmentalimpact; supervised classification; Remote sensing.
\end{abstract}




\section{Introdução}

$\mathrm{O}$ uso e cobertura da terra, de acordo com Food and Agriculture Organization of the United Nations (FAO, 1999), compreende as diferentes estruturas modeladas por meio de forças de ordem natural e antropogênicas. No que se refere à ação humana, as atividades inerentes ao processo de produção e reprodução do espaço geográfico promovem significativas transformações cujo objetivo central consiste em produzir um ambiente que melhor atenda as demandas socioeconômicas e culturais.

No último século, a crescente preocupação com o planejamento territorial e as questões ambientais, decorrentes principalmente das mudanças nouso e cobertura da terra, impulsionaram uma série de debates, que perpassam por problemas associados à desertificação de ambientes áridos e semiáridos, mudanças climáticas, disponibilidade de água para uso difuso, entre outros. Os problemas supracitados decorrem, essencialmente, das práticas de desmatamento, que são empreendidas para conversão de florestas naturais em áreas de pastagem, agricultura, ampliação do perímetro urbano e produção de lenha (Bai, Ochuodho E Yang 2019; Gabiri Et Al., 2019; Ridwansyah et al., 2020).

Nesse sentido, observou-se, então, a necessidade de desenvolver tecnologias capazes de representar a superfície e as alterações espaciais e temporais no uso e cobertura da terra, para diferentes escalas geográficas e cartográficas. Um dos principais mecanismos desenvolvidos para essa finalidade foi a obtenção de imagens de satélites orbitais por meio ou através da utilização de técnicas de sensoriamento remoto.

A maior difusão de imagens de satélite se deu a partir da consolidação do programa Landsat, iniciado por volta da década de 1970. Com a finalidade principal de auxiliar no monitoramento territorial, a série foi continuada e, por essa razão, um robusto banco de imagens foi construído e disponibilizado gratuitamente. Além do fácil acesso ao produ to e as longas séries históricas que se dispõe, outra grande vantagem, que tornou o uso dos produtos Landsat bastante difundidos, foram sua resolução espacial (30 m) e temporal (16 dias), que of erecem boa cobertura da superfície (Novo, 2008). Os avanços com os satélites Landsat tem procurado melhorar a resolução espacial e temporal que podese chegar a oito dias quando intercalados com os diferentes satélites de sua série.
A necessidade de oferecer respostas para os mais variados problemas ambientais fezdosprodutos da série Landsat um dos mais utilizados para o monitoramento e gestão ambiental e diferentes unidades de observação. Atualmente, inúmeros trabalhos foram desenvolvidos, com diferentes abordagens, que vão desde as investigações dos impactos das mudanças no uso e cobertura da terra sobre os recursos hídricos, à questões climáticas e de produtividade agrícola (Silva et al., 2013; Machado et al., 2014; Bezerra et al., (2019); Allam; Bakr; Elbably, 2019; Desta; Fetene, 2020; Murad; Pearse, 2018; Shi etal., 2019; Wang, Q.etal., 2020; Lacerda et al., 2020; Lima et al., 2021; Galvincio e Luz, 2021).

Em geral, a produção de mapas de uso e cobertura da terra se dão por meio da classificação de imagens orbitais, que pode obedecer ao método supervisionado e não supervisionado. No método supervisionado, são utilizados dados obtidos em campo, que podem ser empregados tanto na etapa de classificação quanto na de validação do produto, a fim de avaliar o grau de precisão do mapeamento. Entretanto, o levantamento dessas informações não é uma tarefa relativamente simples ou possível, seja pela dificuldade de acesso às áreas, tempo demandado ou limitação de recursos financeiros (Wulder et al., 2006).

No sentido de contornaro referidoproblema, autores como Fritz et al.(2009), sugeriram o uso de imagens de satélites de alta resolução espacial, disponibilizadas gratuitamente em programas como o Google Earth Pro. Essas imagens, em geral, são integradas ao referido software e possuem detalhamento superficial relativamente alto para que o observador possa distinguir os alvos dispostos na superfície.

Entre as primeiras aplicações de relevância, destaca-se o estudo conduzido por Biradar et al.(2009). Um dos principais objetivos da pesquisa foi realizar um mapeamento das áreas de agricultura de sequeiro em escala global. Por meio de imagens orbitais disponíveis no banco de dados do Google Earth, foi realizada uma amostragem, para a qual foram demarcados 1009 pontos, distribuídos aleatoriamente, e outros 915 obtidos em campo. O teste de acurácia do mapeamento, realizado por meio do teste de Kappa, variaram entre 92 e 98\%, comprovando a eficácia da metodologia para uma grande escala geográfica. 
A metodologia também foi empregada para melhoramento e atualização de mapas antigos, considerados menos precisos em relação aos que se dispõe atualmente. Pekkarinen Reithmaier e Strobl (2009), por exemplo, classificaram 415 cenas do satélite Landsat 7, para mapeamento florestal de todo o continente europeu. $\mathrm{Na}$ amostragem, os autores utilizaram, em média, 1041 pontos por cena. Os resultados encontrados após o processamento e classificação supervisionada indicaram uma precisão em torno de $88 \%$, com nível de significância estatística $\geq 95 \%$.

Além dos trabalhos supracitados, outras pesquisas, voltadas para diferentes linhas de abordagem, que foram desde o teste da metodologia à análises ambientais foram desenvolvidos e se revelaram exitosos (Clark et al., 2010; Hansen et al., 2014; Jacobson, A. et al., 2015; Redo; Aide; Clark, 2012; Saito et al., 2016).

A robustez dos resultados encontrados a partir dos diferentes estudos citados foi reveladora também de sua versatilidade. Nesse sentido, a possibilidade de aplicações que o método, inicialmente, oferece fez surgir um importante questionamento: esse pode ser utilizado para representações espaço-temporais de áreas semiáridas, a fim de promover investigações sobre as práticas de desmatamento? Esta questão será o ponto de partida para o desenvolvimento da presente pesquisa, que tem como objetivo central analisar as mudanças espaço-temporais do uso e cobertura da terra na bacia hidrográfica do rio Pontal, localizada no estado de Pernambuco.

A bacia hidrográfica do rio Pontal fica localizada em área do semiárido de Pernambuco com grande desenvolvimento econômico voltado para agricultura irrigada. O grande desenvolvimento econômicose dar devido a disponibilidadehídrica do rio São Francisco que faz com que uma região semiárida se torne uma das maiores produtoras de uva e vinho do Brasil. Acredita-se que esta seja uma bacia que possua grandes transformações ao longo dos anos e pode ser monitorada o seu uso e ocupação usando a metodologias e ferramentas propostas neste estudo. Além disso, esse artigo tenta contribuir para os seguintes objetivos do desenvolvimento sustentável, fome zero e agricultura sustentável, consumo e produção sustentável, ação contra as mudanças do clima e vida terrestre. Quando ele tenta auxiliar no monitoramento do uso e ocupação do solo e possa deix ar informações importantes para a gestão da bacia hidrográfica em estudo.

\section{Material e métodos}

\section{Localização e caracterização da área de estudo}

A área de estudo corresponde a bacia hidrográfica do rio Pontal, também denominada Unidade de Planejamento Hídrico UP13 (Figura 1). Está localizada no extremo oeste do estado de Pernambuco, entre as coordenadas geográficas $08^{\circ}$ 19' $00^{\prime \prime}$ e $09^{\circ} 13^{\prime} 24^{\prime \prime}$ de latitude sul, e $40^{\circ} 11^{\prime} 42^{\prime \prime}$ e $41^{\circ} 20^{\prime} 39^{\prime}$ ' de longitude oeste. A bacia possui área de drenagem correspondente a $6.015,33 \mathrm{~km}^{2}$, com direção que predomina no sentido noroeste-sudeste, seguindo até sua foz, na margem esquerda do rio São Francisco. O clima predominante na região é o tropical semiárido, segundo a classificação de Köppen. A precipitação é bastante escassa, e se concentra nos meses de dezembro a abril, ocorrendo sob influência de sistemas atmosféricos dinâmicos, entre os quais pode-se destacar, a Zona de Convergência Intertropical (ZCIT) (Ferreira, et al., 2017; Silva et al., 2016)

A vegetação predominante é do tipo xerófila, com a maior presença de SavanaEstépica Arborizada e Florestada. A bacia hidrográfica do rio Pontal é composta por quatro municípios, sendo eles, Afrânio, em sua totalidade, Dormentes, Lagoa Grande e Petrolina. Os municípios pertencentes a região têm sua base econômica fortemente atrelada ao setor agrícola, o polo fruticultor de Petrolina se destaca nesse sentido, com produção voltada para exportação (Agência Pernambucana de Água e Clima-APAC, 2018; Silva et al., 2016). 

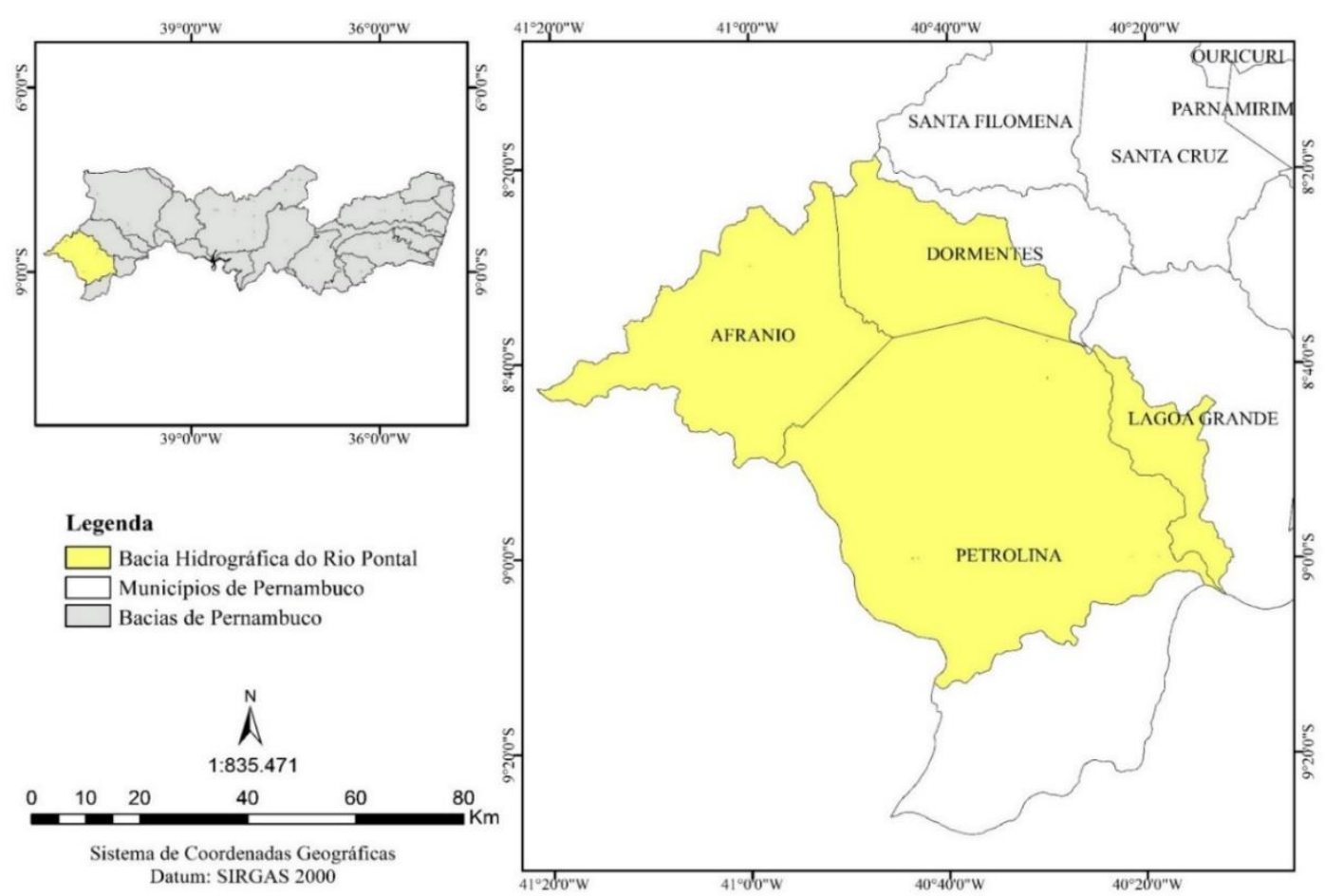

Figura 1. Localização geográfica da bacia hidrográfica do rio Pontal - PE.

\section{Procedimentos metodológicos}

Para determinar as mudanças espaciais e temporais do uso e cobertura da terra na bacia hidrográfica do rio Pontal, foram utilizadas imagens dos satélites Landsat 5 e 8 . As cenas utilizadas do satélite Landsat 5, correspondem às órbitas e pontos 218/66 (02/10/1992) e 217/66(24/08/1992). Em relação às imagens Landsat 8 utilizadas, as duas cenas foram das órbitas e pontos 218/66 $(05 / 11 / 2020)$ e $217 / 66(08 / 10 / 2020)$. As imagens foram obtidas gratuitamente junto ao site da U.S.Geological Survey (USGS). Todas possuem resolução espacial de 30 metros e resolução radiométrica de 8 bits para as imagens Landsat 5 e 16 bits para as cenas Landsat 8. A escolha dessas datas se deram com intuito de avaliar as mudanças dos últimos 30 anos e que pudéssemos contar com imagens de satélites de qualidade.

Após aquisição dos produtos, realizou-se empilhamento das bandas das imagens. Posteriormente, foram realizados os cálculos da radiância e reflctância utilizando como base os artigos de Machadoet al., (2014); Silva et al., (2013); Lacerda et al., (2020); Lima et al., (2021); Galvincio e Luz (2021):
Landsat 5

A radiância espectral de cada banda ( $\mathrm{L} \lambda \mathrm{i})$ representa a energia solar refletida por cada pixel por unidade de área, de tempo, de ângulo sólido e de comprimento de onda, medida a nível do satélite Landsat (aproximadamente $705 \mathrm{~km}$ de altura), para as bandas $1,2,3,4,5$ e 7; para a banda 6 , essa radiância representa a energia emitida por cada pixel e pode ser obtida pela Equação 1:

$L_{\lambda i}=\alpha_{t}+\frac{b_{t}-\alpha_{t}}{255} N D$

Onde:

a e b são as radiâncias espectrais mínima e máxima (Wm-2 sr-1 $\mu \mathrm{m}-1)$, detectadas pelo TM - Landsat 5; ND é a intensidade do pixel (número inteiro compreendido entre 0 e 255, inclusive) e $\mathrm{i}$ corresponde às bandas do TM - Landsat 5 .

A reflectância monocromáticade cadabanda ( $\rho \lambda \mathrm{i})$, definida como sendo a razão entre o fluxo de radiação solar refletido e o fluxo de radiação solar incidente, foi obtida segundo a Equação 2: 
$\rho_{\lambda i}=\frac{\pi \cdot L_{\lambda i}}{k_{\lambda i} \cdot \operatorname{cosZ} \cdot d_{r}}$

Onde:

L $\lambda$ i é a radiância espectral de cada banda, $k \lambda i$ é a irradiância solar espectral de cada banda no topo da atmosfera (Wm-2 $\mu \mathrm{m}-1)$, Z é o ângulo zenital solar e dr é o quadrado da razão entre a distância média Terra-Sol (ro) e a distância Terra-Sol (r) em dado dia do ano (Equação 3):

$d_{r}=1+0,033 \cos \left(\frac{D S A 2 \pi}{365}\right)$

Onde:

DSA representa o dia sequencial do ano e o argumento da função cos está em radianos (Equação 4). $\mathrm{O}$ valor médio anual de dr é igual a 1,00 e o mesmo varia de 0,97 a 1,03, aproximadamente. Quando a área de estudo tem pequena, ou mesmo, declividade nula, o cosseno do ângulo de incidência da radiação solar é simplesmente obtido a partir do ângulo de elevação do Sol - E, que se encontram no metadados da imagem, ou seja:

$\cos Z=\cos \left(\frac{\pi}{2}-E\right)=\operatorname{sen}(E)$

em que o argumento do cos está em radiano.

\subsubsection{Landsat 8}

A reflectância que consiste no cálculo entre o fluxo de radiação solar refletido e o fluxo de radiação solar incidente, e é representado pela Equação 5:

$r b=\frac{\left(A d d_{r e f, b}+M u l t_{r e f, b} N D_{b}\right)}{\operatorname{cosZ} \cdot d_{r}}$

Em que:

Add corresponde aof ator aditivo de reescalonamento para cada banda, disponível no arquivo metadados da imagem, assim como o Mult que corresponde ao fator multiplicativo de reescalonamento para cada banda (Tabela 1). ND representa os valores de número digital da imagem, $\mathrm{Z}$ é o ângulo zenital solar(calculado a partir de $\theta \mathrm{SZ}=90^{\circ}-\theta \mathrm{SE}$ ), o ângulo de elevação solar ( $\theta$ SE) e também pode ser encontrado no arquivo metadados da imagem. $\mathrm{O}$ dr corresponde ao quadrado da razão entre a distância média Terra-Sol e a distância Terra-Sol em dado dia do ano, e pode ser calculado através da Equação 6:

$\mathrm{d}_{r}=\left(\frac{1}{d_{T R}}\right)$

Tabela 1. Termos aditivos e multiplicativos para o cômputo da reflectância.

\begin{tabular}{ccr}
\hline BANDA & ADD_ref & MULT_ref $_{\mathrm{b}}$ \\
\hline 2 & -0.100000 & 0,00002 \\
3 & -0.100000 & 0,00002 \\
4 & -0.100000 & 0,00002 \\
5 & -0.100000 & 0,00002 \\
6 & -0.100000 & 0,00002 \\
7 & -0.100000 & 0,00002 \\
\hline
\end{tabular}

Fonte: Metadados das imagens OLI: órbita 218, ponto $66(05 / 11 / 2020)$ e órbita 217 , ponto 66 (08/10/2020).

\section{Classificação supervisionada}

Após processamento das imagens até a etapa de refletância, foi realizado o procedimento de classificação supervisionada das imagens. A classificação foi baseada no conjunto de imagens de alta resolução espacial agregadas ao banco de dados da plataforma Google Earth Pro (GEP). Esse método, permite que o usuário identifique à vista desarmada as classes predominantes na superfície da área de estudo e a distribuição dessas no espaço com bom nível de detalhamento. Maiores informações sobre o método proposto podem ser encontradas no estudo desenvolvido por Fritz et al. (2009).

Para extrair as informações, foi gerada em ambiente SIG, uma nuvem de pontos com cerca de 1.200, no formato shapefile. Posteriormente, o arquivo relacionado a esse atributo foi importado para dentro da plataforma GEP, conforme pode ser observado na Figura 3. 


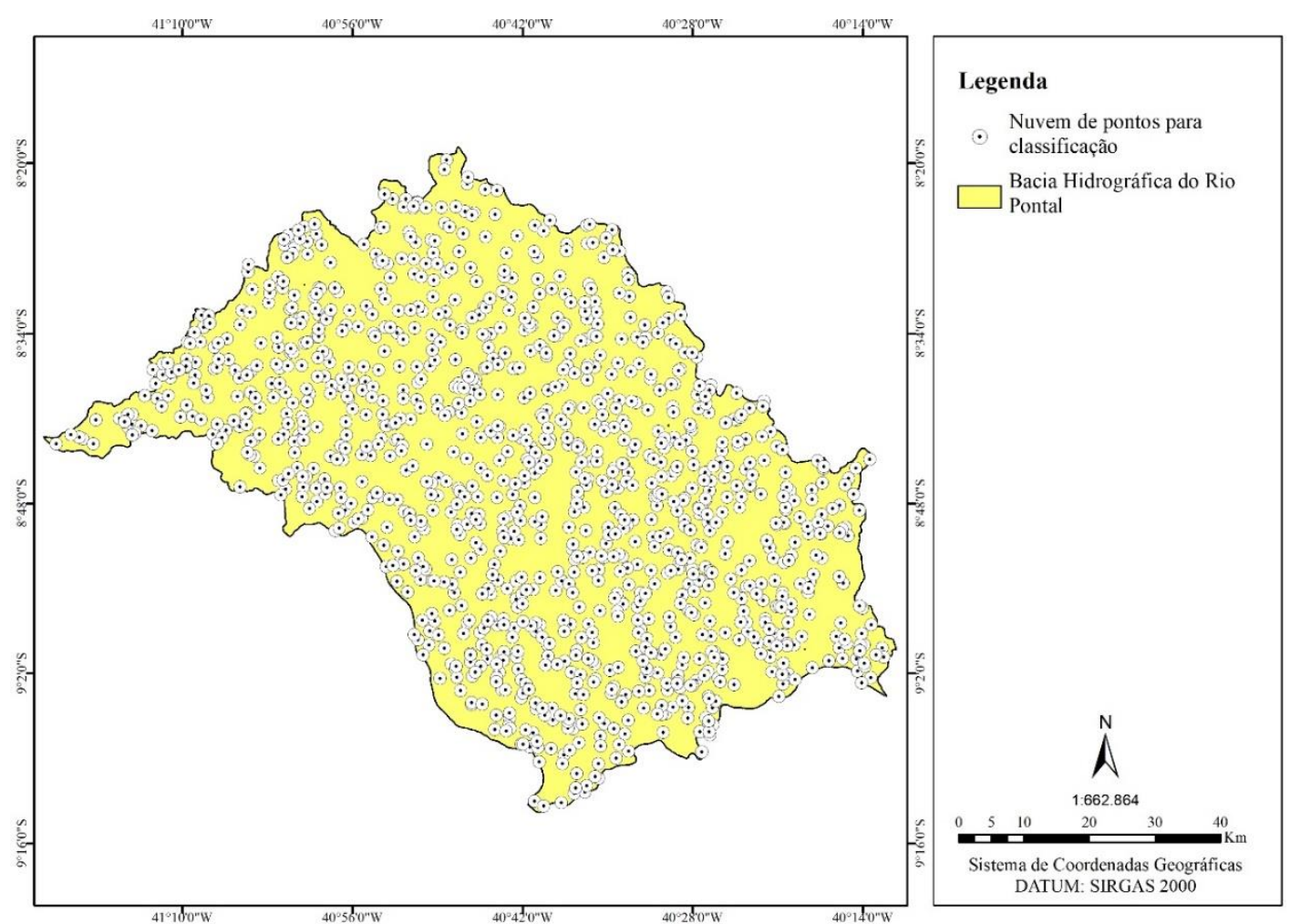

Figura 2. Nuvem com 1200 pontos distribuídos sobre a bacia hidrográfica do rio Pontal.

O procedimento seguinte consistiu em ampliar cada um dos pontos (Figura 4), com auxîlio da ferramenta zoom do GEP, o que veio a permitir a interpretação visual e posterior distinção das classes. A cada cobertura interpretada foi atribuído um valor numérico que representou a categoria de uso (1 Água, 2 - Solos Expostos e Agropecuária, 3 - Savana Estépica Florestada, 4 - Savana Estépica Arborizada e 5 - Agricultura Irrigada).

Algumas imagens do Google Earth são temporalmente defasadas (distantes do ano de referência da imagem empregada para classificação) ou possuem resolução espacial com nível de detalhe que não permite a fácil distinção e interpretação dos alvos da superfície. Por essa razão, alguns pontos foram sumariamente descartados quando observado que correspondiam a uma das realidades técnicas mencionadas. Ao todo, foram excluídos 94 pontos para a nuvem correspondente a imagem Landsat 5 e 55 para a Landsat 8.

A etapa seguinte consistiu em gerar as assinaturas espectrais das classes de uso e cobertura da terra a partir das imagens de reflectância processadas dos satélites Landsat 5 e 8 . Cada uma dessas foi relacionada aos respectivos códigos que representaram cada uma das classes identificadas durante a etapa de classificação. 

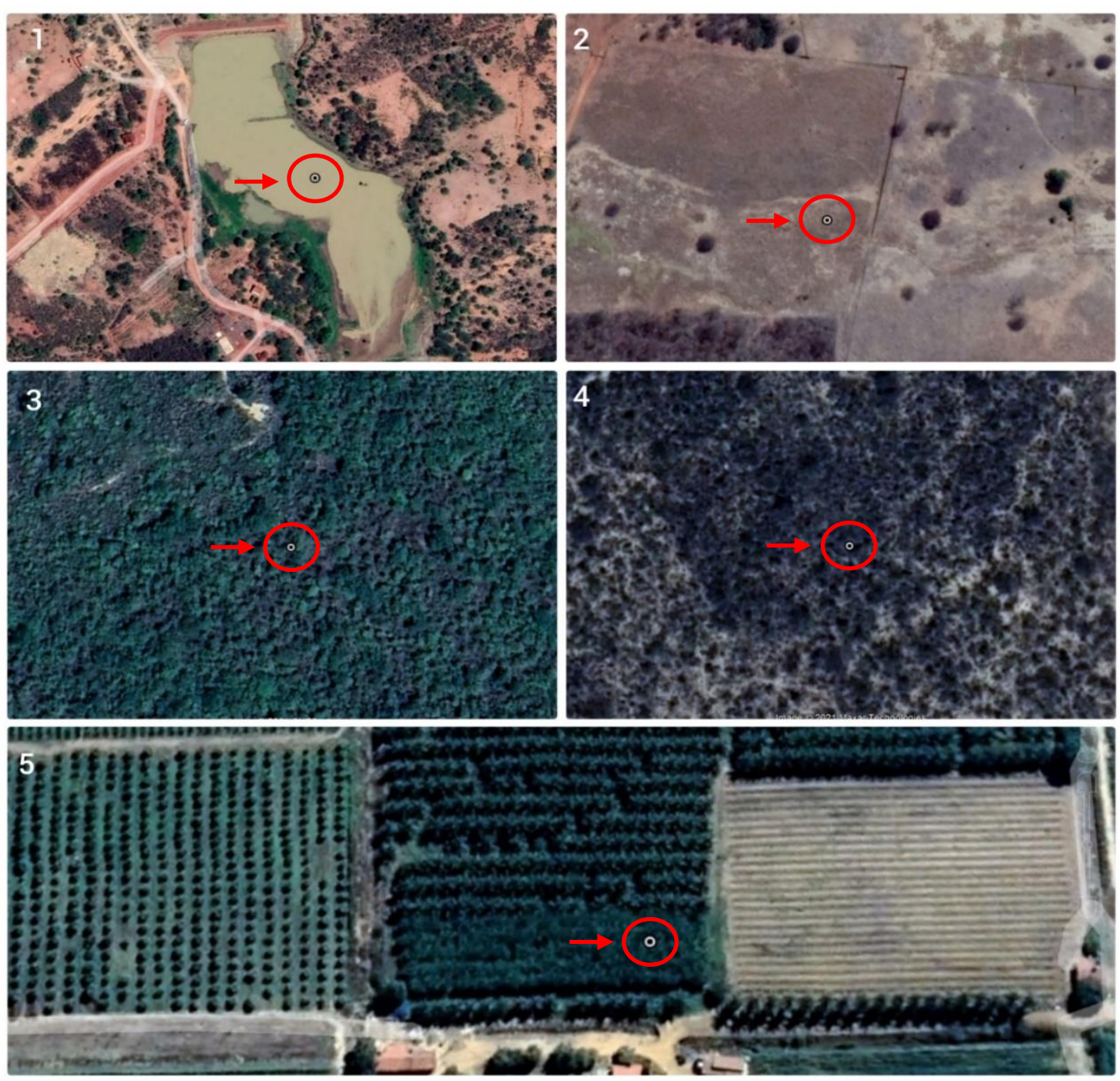

Figura 4. Classes de uso e cobertura da terra encontrados na área de estudo. 4.1: Corpos Hídricos; 4.2: Solos Expostos e Agropecuária; 4.3:Savana Estépica Florestada; 4.4: Savana Estépica Arborizada, 4.5:Agricultura Irrigada.

O referido procedimento foi realizado com auxílio da ferramenta Create Signature do ArGis.

Por fim, as imagens foram submetidas ao processo de classificação supervisionada, que é realizado por meio do algoritmo Maximum Likelihood. Assim, as informações correspondentes às assinaturas espectrais foram relacionadas com as suas respectivas imagens e o processamento foi concluído.

O mapeamento deuso e ocupação do solo foi comparado com o realizado por Miranda et al., (2017) em que ele avaliou as mudanças no uso e ocupação do solo da bacia do Pontal de 1975 a 2013. Os resultados de Miranda estão disponível no SUPerSistema de Unidades de Respostas Hidrológicas para
Pernambuco, site https://super.hawqs.tamu.edu/\#/admin/users e no repositório da UFPE-Universidade Federal de Pernambuco,

https://repositorio.ufpe.br/bitstream/123456789/251 88/1/TESE\%20Rodrigo\%20de\%20Queiroga\%20Mi randa.pdf. Considerou como referência real os dados da tese de Miranda e sistema SUPer.

\section{Resultados e discussão}

Algumas das imagens que integram o banco de dados do Google Earth Pro apresentaram resolução espacial inferior ou equivalente dos satélites Landsat 5 e 8 para algumas localidades da 
bacia hidrográfica do rio Pontal. Verificou-se também significativa defasagem temporal para alguns pontos. Ou seja, situações em que a imagem do banco de dados apresentava mais de três anos de diferença em relação às utilizadas no presente estudo. Ao todo, 94 pontos amostrais sobrepuseram imagens que apresentaram uma das características mencionadas anteriormente, o que culminou com a exclusão desses. Desse total, 89 corresponderam ao cenário gerado a partir das imagens do satélite Landsat 5 e os demais para o Landsat 8.

Quanto ao produto gerado a partir da classificação supervisionada, este pode ser observado na Figura 7. O mapeamento desse tipo de atributo para regiões de clima semiárido não é um procedimento simples, dadas as características do bioma dominante na região (caatinga). Batista, Santos, R. L. (2011) destacam que a sazonalidade da vegetação responde principalmente a variação da precipitação pluviométrica. Nesse sentido, durante o período seco, as espécies perdem a folhagem para reduzir o estresse luminoso e a consequente perda de água para a atmosfera. Esse mecanismo de defesa é vital para a sobrevivência das plantas durante os longos períodos de estiagem.

Por outro lado, a estação seca na região faz aumentar o ef eito do solo sobre a resposta espectral captada pelos sensores dos satélites imagiadores.
Essa condição pode resultar em mapeamentos imprecisos, com áreas de solos expostos mais extensas, principalmente em ocasiões para as quais não é possível realizar um trabalho de campo. Entretanto, a partir do método utilizado para o desenvolvimento do presente estudo, não houve dificuldade para identificar as áreas vegetadas mesmo durante o período seco. As respostas espectrais para áreas sob essas condições, ainda que se aproximem do que frequentemente é encontrado em áreas de solos expostos, são significativamente diferentes para que o algoritmo utilizado possa classificá-las como área vegetada.

O método revelou-se eficiente na detecção das diferentes classes encontradas na área de estudo, assim como na variação espacial e temporal para o período analisado. Isso também foi observado em trabalhos desenvolvidos por (Desta; Fetene, 2020; Jacobson, A. et al., 2015; Zaidi et al., 2017). Por outro lado, um estudo que é mais próximo da realidade físico-natural da bacia hidrográfica do rio Pontal foi o conduzido por Gomes da Silva, M. L. et al., 2016. Ao utilizar a mesma técnica, porém com uma quantidade de pontos, proporcionalmente infeior, os autores não só identificaram os diferentes tipos de classes de uso e cobertura da terra e a variação espacial e temporal da vegetação natural e agrícola.

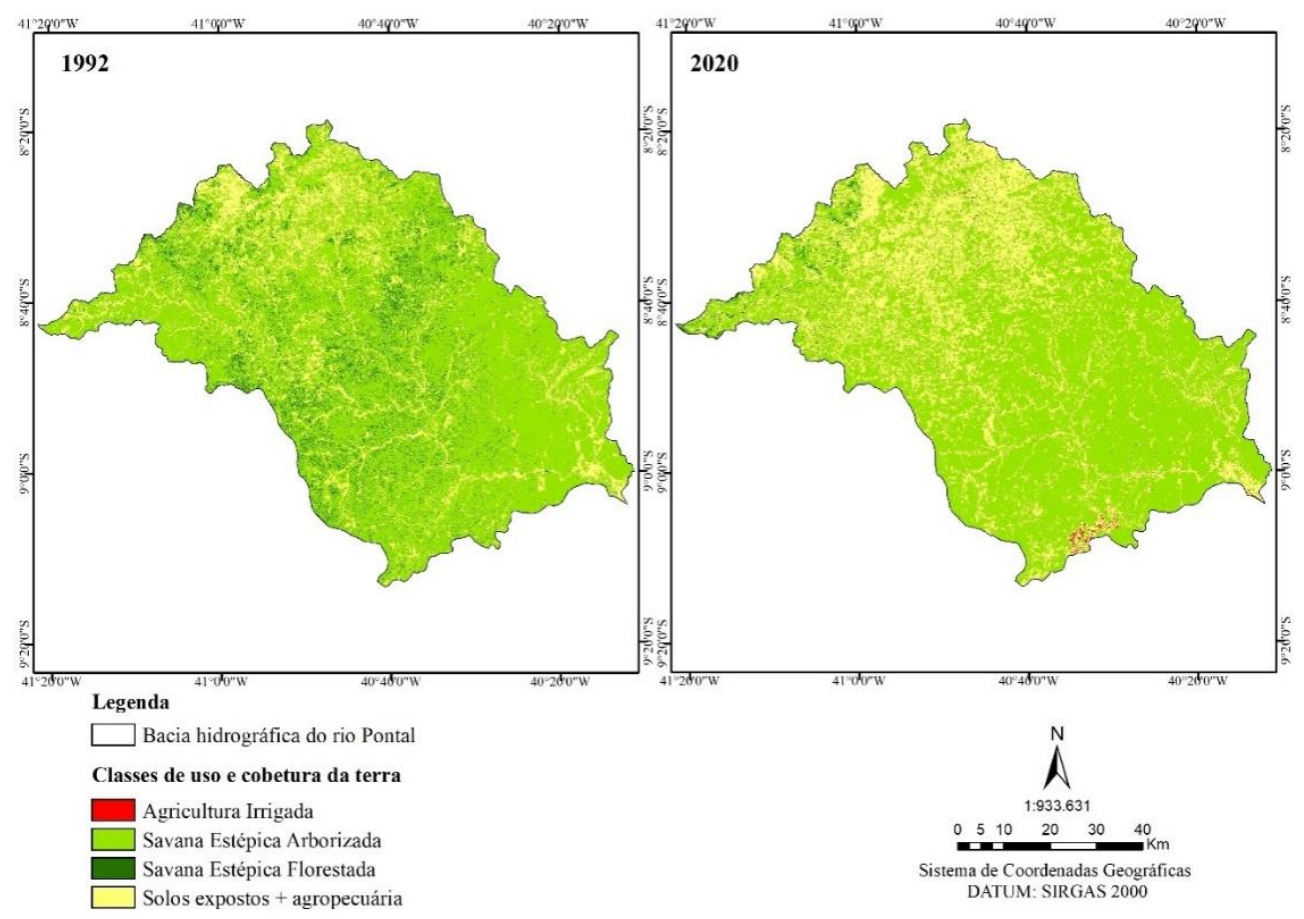

Figura 5. Mapeamento do uso e cobertura da terra na bacia hidrográfica do rio Pontal para os anos de 1992 e 2020. 
Quanto as classes de uso e cobertura encontradas, a mais expressiva em termos de área corresponde à Savana Estépica Arborizada. Esse tipo de vegetação se caracteriza por apresentar baixo porte e fisionomia do tipo arbustiva. Sob determinadas condições, a depender do tipo de solo, disponibilidade de água e nível de conservação, podem apresentar florestas adensadas ou vastasáreas com extensas clareiras, onde os indivíduos apresentam espaçamento importante entre si, produzindo maior exposição dos solos. Regiões cuja predominância de espaçamento entre as espécies vegetais é mais comum, apresentam, de modo geral, maior suscetibilidade dos solos aos agentes extemos e, por essa razão, são mais vulneráveis.

$\mathrm{Na}$ bacia do rio Pontal, esse tipo de vegetação é bem distribuído ao longo da superfície, porém, até o ano de 1992, apresentava menor fragmentação nas regiões centroe montante da bacia. No referido ano, a área ocupada pela Savana Estépica Arborizada era de cerca de $74 \%$, com maior concentração à jusante. Por outro lado, em 2020, a área ocupada foi reduzida para cerca de $67 \%$, o que representou uma taxa de degradação em torno de $0,25 \%$ ao ano. Esse resultado corrobora o de Miranda (2017), Miranda ela t., (2018) que mostra uma maior fragmentação da caatinga e diminuição de área total.

A segunda classe mais representativa referese aos Solos Expostos + Agropecuária. Ambas foram integradas devido à dificuldade de separação a partir de imagens orbitais. Esse procedimento só seria possível mediante um minucioso trabalho de campo. Porém, além da inviabilidade técnico-operacional, o proposto para esse estudo, atende ao objetivo central, que é identificar as áreas degradadas e as que apresentam vegetação nativa a partir de um método de classificação alternativo.

Isso posto, pôde-se comprovar que houve um intenso processo de degradação das vegetações nativas na área de estudo, visto que se identificou significativo crescimento da classe de Solos Expostos + Agropecuária, que ocupava $18 \%$ da superfície total em 1992 e passou para cerca de $31 \%$ em 2020. Ou seja, uma taxa de crescimento de $0,46 \%$ ao ano.

A última classe encontrada é a Savana Estépica Florestada. Apesar de ocupar apenas 7,7\% da área de estudo, esse tipo de vegetação, foi, proporcionalmente, a mais degradada, visto que em 2020 a sua ocupação reduzida para $0,9 \%$.

Impacto do uso e ocupação do solo no ciclo hidrológico

A Figura 6 mostra o balanço hídrico médio anual da bacia do Pontal de 1961-2021. Esse balanço hídrico computa todas as mudanças de uso e ocupação do solo ocorrida na bacia. Dessas mudanças a bacia hidrográfica do Pontal conta em 1992, Figura 7, com uma média anual de $50,51 \mathrm{~mm}$ de perda de água do solo através da evaporação do solo e de uma infiltração/percolação de $77,6 \mathrm{~mm}$. Comparando com o ano de 2021, Figura 6, destacase que ocorreu uma diminuição da perda de água no solo de $25 \%$, passando a perder $37,96 \mathrm{~mm}$ e uma diminuição de $31 \%$ de infiltração/percolação. Sabese que o desmatamento diminui a infiltração de água no solo e c tipo de impacto no balanço hídrico tem relação com o desmatamento. É importante destacar que ocorreu uma diminuição das chuvas de $11 \%$ que pode estar relacionada as mudanças climáticas globais. Porém, é confiável afirmar que as mudanças no uso e ocupação do solo contribuíram para a diminuição de $31 \%$ da infiltração de água no solo e consequentemente diminuição do escoamento lateral. Sabe-se que no semiárido a água armazenada no solo tem grande importância para manutenção dos ecossistemas naturais. Assim, é urgente uma ação que possa melhor monitorar e gerenciar a bacia do Pontal. Destaca-se também ocorreu uma diminuição do escoamento superficial. Esse impacto está relacionado a diminuição das chuvas na região em consequência das mudanças climáticas globais. Mas, é importante destacar que para ocorrer escoamento superficial quando da ocorrência de chuvas de pouca intensidade e longa duração é necessário a saturação do solo. Quando ocorre o desmatamento há uma diminuição da saturação do solo mesmo quando da ocorrência de chuvas de pouca intensidade. Pois, devido a inexistência de plantas/arvores a água escoa livremente e diminui a infiltração de água no solo. Destaca-se que a bacia do Pontal possui hoje menos água disponível no seu balanço hídrico para os diferentes usos e sustentabilidade ambiental devido as mudanças climáticas globais e mudanças no uso e ocupação do solo. Galvincio e Luz (2021) tem 
simularam os impactos das mudanças climáticas sobre a bacia do Pontal até 2027 e afirmaram que ocorrerá uma diminuição de $15 \%$ na precipitação na bacia até esta data. Assim, é importante destacar a necessidade de revitalização dabacia noque se refere a reflorestamento e preservação da vegetação natural ainda existente. Essa necessidade se dá em função da sustentabilidade econômica e ambiental da área em estudo. Galvincio (2021) ainda afirma que situação semelhante a bacia do Pontal pode ocorrer nas bacias que são banhadas pelos municípios de Belém do São Francisco e Serra Talhada.

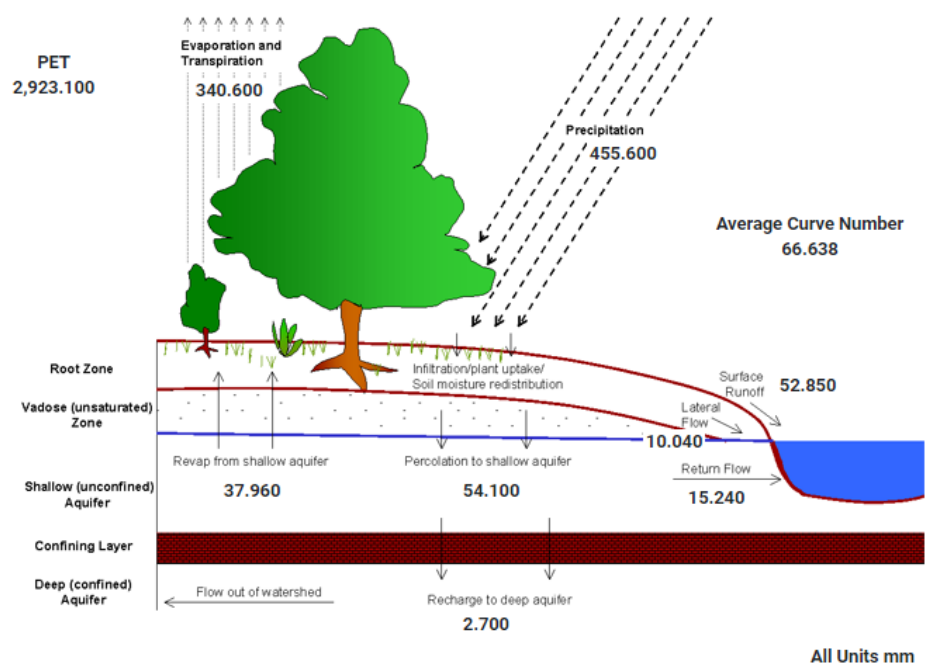

Figura 6- Balanço hídrico médio anual da bacia do Pontal de 1961-2021.

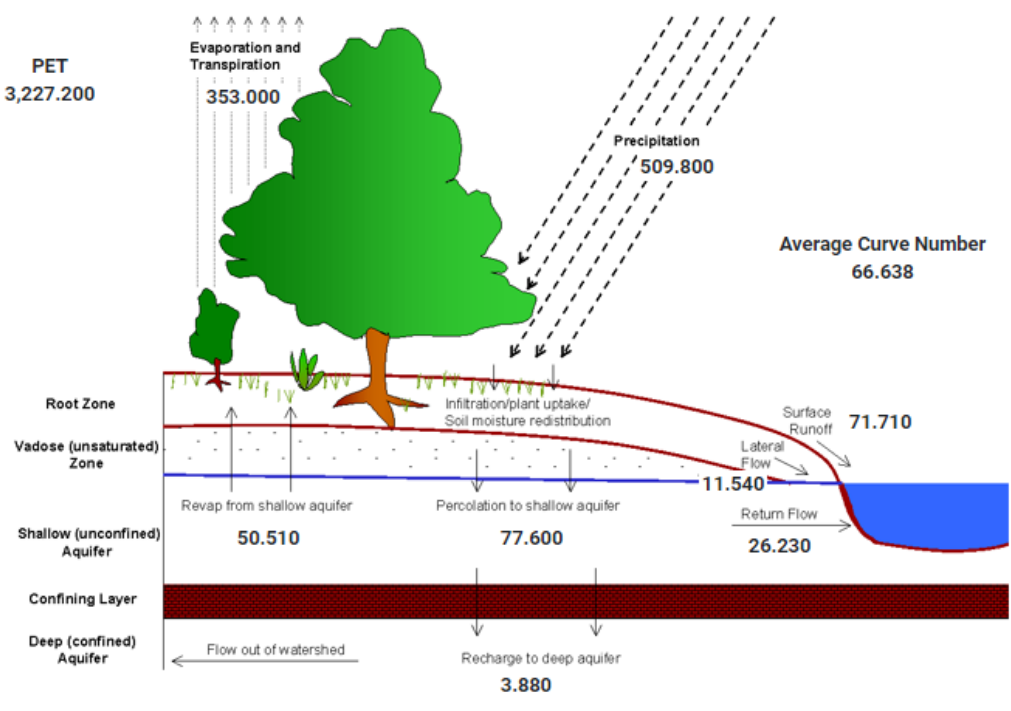

Figura 7- Balanço hídrico médio anual da bacia do Pontal de 1961-1992.

\section{Conclusões}

A bacia do Pontal possui hoje menos água disponível no seu balanço hídrico para os diferentes usos e sustentabilidade ambiental devido as mudanças climáticas globais e mudanças no uso e ocupação do solo.

As técnicas empregadas para o mapeamento do uso e cobertura da bacia hidrográfica do rio Pontal 
se mostraram consistentes no sentido de representar a variação espacial e temporal da vegetação na referida unidade. O método foi capaz de identificar as mudanças espaço-temporais, independentemente da escala geográfica da classe de uso e cobertura.

A área estudada está em avançado processo de degradação de sua vegetação nativa. Isso se deve principalmente à expansão das áreas de agricultura e pecuária, principal modelo de desenvolvimento socioeconômico observado na região. Por outro lado, é importante destacar também que os perímetros irrigados também possuem sua contribuição para o desmatamento observado, porém, em escala mais restrita quando comparado às demais.

Vale ressaltar que o Semiárido é uma região marcada pela escassez hídrica e enf renta problemas associados à desertificação e mudanças climáticas. Nesse sentido, a remoção da cobertura vegetal figura como um agravante para essas questões.

Por fim, conclui-se que novas abordagens para análises devem ser desenvolvidas no sentido de oferecer respostas para as questões de ordem socioambiental na região. Assim, o presente estudo pode ser utilizado como ponto de partida ou referência para pesquisas cujo uso e cobertura da terra seja um dos atributos ambientais considerados nas investigações.

Ainda se destaca que este estudo possa ser utilizado como apoio ao monitoramento e gestão da bacia do Pontal devido a necessidade de um gerenciamento ambiental mais rigoroso no âmbito da bacia estudada, principalmente no que se refere à tomada de decisão quanto a outorga de água para implantação ou aumento de áreas de agricultura irrigada, para que não ocorram prejuízos irrecuperáveis e significativos para a população que é beneficiada pela bacia do Pontal. Visto que o desmatamento descontrolado na bacia influi negativamente nas condições ambientais dessa bacia.

\section{Agradecimentos}

À Fundação de Amparo à Ciência e Tecnologia de Pernambuco (FACEPE), pelo apoio financeiro na conceção de bolsa de doutorado. À Universidade Federal de Pernambuco (UFPE), pela estrutura. Ao CNPq- Conselho Nacional de Desenvolvimento Científico e Tecnológico pelas bolsas de produtividade de pesquisa do segundo e terceiro autor.

\section{Referências}

Allam, M.; Bakr, N.; Elbably, W. 2019. Multitemporal assessment of land use/land cover change in arid region based on landsat satellite imagery: Case study in Fayoum Region, Egypt. Remote Sensing Applications: Society and Environment,. 14, 8-19. Disponível em: <https://doi.org/10.1016/j.rsase.2019.02.002>.

Bai, Y.; Ochuodho, T. O.; Yang, J. Impact of land use and climate change on water-related ecosystem services in Kentucky, USA. Ecological Indicators, 2019. v. 102, n. July 2018, p. 51-64. Disponível em: <https://doi.org/10.1016/j.ecolind.2019.01.079>.

Batista, J. L. O.; Santos, R. L. 2011. Análise da dinâmica do usoe ocupação da terra em pequenos municípios baianos do semi-árido brasileiro: $\mathrm{O}$ caso de Teofilândia. Revista de Geografia Norte Grande, 49, 139-155.

Bezerra, J., Andrade, J., Melo, F., Vigoderis, R., Galvíncio, J., Souza, W. 2019. Degradation of the Vila Maria Spring in Garanhuns-PE. Joumal of Hyperspectral Remote Sensing, 9(6), 320-329. doi:10.29150/jhrs.v9.6.p320-329.

Biradar, C. M. et al. 2009. A global map of rainfed cropland areas (GMRCA) at the end of last millennium using remote sensing. International Journal of Applied Earth Observation and Geoinformation, 11, 114-129.

Clark, M. L. et al. 2010. A scalable approach to mapping annual land cover at $250 \mathrm{~m}$ using MODIS time series data: A case study in the Dry Chaco ecoregion of South America. Remote Sensing of Environment, 114, 2816-2832. Disponível em: <http://dx.doi.org/10.1016/j.rse.2010.07.001>.

Desta, H.; Fetene, A. 2020. Land-use and land-cover change in Lake Ziway watershed of the Ethiopian Central Rift Valley Region and its environmental impacts. Land Use Policy, 96, 104682. Disponível em: <https://doi.org/10.1016/j.landusepol.2020.1046 $82>$.

Ferreira, P. Dos S. et al. 2017. Análise da suscetibilidade a desertificação na bacia hidrográfica do rio pontal - Pernambuco - Brasil. Investigaciones Geográficas, 53, 37.

Fritz, S. et al. 2009. Geo-wiki.org: The use of crowdsourcing to improve global land cover. Remote Sensing, 1, 345-354. 
Fernandes, J. G.; Freire, M. B. G. dos S.; Cunha, J. C.; Galvíncio, J. D.; Corrêa, M. M.; Santos, P. R. dos. 2009. Qualidade físico-química das águas utilizadas no Perímetro Irrigado Cachoeira II, Serra Talhada, Pernambuco. Agrária (Recife. Online), 4, 27-34.

Galvíncio, J. (2021). Impacto do aumento de CO2 nas Precipitações do estado de Pernambuco. Revista Brasileira de Geografia Física, 14, 18281839. doi:10.26848/rbgf.v14.3.p1828-1839.

Galvíncio, J.D., Mendes, S.M., Souza, W.M., De Moura, M.S.B., Santos, W. 2020. Linear correlation between rainfall and leaf area index of the Caatinga biome | Correlação linear entre a precipitação e o Índice de Área Foliar do bioma Caatinga. Revista Brasileira de Geografia Fisica 13, 3304-3314.

Gabiri, G. et al. 2019. Modelling the impact of land use management on water resources in a tropical inland valley catchment of central Uganda, East Africa. Science of the Total Environment, 653, 1052-1066. Disponível em: <https://doi.org/10.1016/j.scitotenv.2018.10.430 $>$.

Galvincio, J. D. Luz, G. G. 2021. Desenvolvimento de Modelo que Estima o Impacto do $\mathrm{CO} 2$ Atmosférico nas Precipitações do Estado de Pernambuco, utilizando ARIMA. Revista Brasileira de Geografia Física, 14, 1840-1851.

Hansen, M. C. et al. 2014. Monitoring conterminous United States (CONUS) land cover change with Web-Enabled Landsat Data (WELD). Remote Sensing of Environment, 140, 466-484.

Jacobson, A. et al. 2015. A novel approach to mapping land conversion using Google Earth with an application to East Africa. Environmental Modelling \& Software, 72, 1-9. Disponível em: $<$ http://www.sciencedirect.com/science/article/pi i/S1364815215001747>.

Lacerda, A.C., Galvincio, J.D., Morais, Y.C.B., Pimentel, R.M.M., de Moura, M.S.B. 2020. Edapho-topo-climatic influence on gross primary production in semi-arid | Influência topoedafoclimática na produção primáriabruta no semiárido. Revista Brasileira de Geografia Fisica, 13, 3119-3135.

Lima, C.E.S.D., Costa, V.S.D.O., Galvíncio, J.D., Silva, R.M.D., Santos, C.A.G. 2021. Assessment of automated evapotranspiration estimates obtained using the GP-SEBAL algorithm for dry forest vegetation (Caatinga) and agricultural areas in the Brazilian semiarid region. Agricultural Water Management, 250, 106863.

Machado, C.C.C.; Silva, B. B. Albuquerque, Manoel B.; Galvíncio, J. D. 2014. Estimativa do Balanço de energia utilizando imagens TM: Landsat 5 e o algoritmo SEBAL no litoral sul de Pernambuco. Revista Brasileira de Meteorologia (Impresso), 29, 55-67.

Miranda, R. G. 2017. Avaliação Integrada Da Variação Espacial E Temporal Do Balanço Hídrico $\mathrm{Na}$ Caatinga. 123p. https://repositorio.ufpe.br/bitstream/123456789/ 25188/1/TESE\%20Rodrigo\%20de\%20Queiroga $\%$ 20Miranda.pdf

Miranda, R. Q., Galvincio, J.D., Morais, I. C. B., Moura, M. S. B., Jones, C. A., Srinivasan, R. 2018. Dry Forest Deforestation Dynamics In Brazil's Pontal Basin. Revista Caatinga 31 https://doi.org/10.1590/1983$21252018 \mathrm{v} 31 \mathrm{n} 215 \mathrm{rc}$

Murad, C. A.; Pearse, J. 2018. Landsat study of deforestation in the Amazon region of Colombia: Departments of Caquetá and Putumayo. Remote Sensing Applications: Society and Environment, 11, 161-171. Disponível em: <https://doi.org/10.1016/j.rsase.2018.07.003>.

Novo, E. M. L. M. 2008. Sensoriamento Remoto: princípios e aplicações. São Paulo.

Pekkarinen, A.; Reithmaier, L.; Strobl, P. 2009. PanEuropeanforest/non-forest mapping with Landsat ETM+ and CORINE Land Cover 2000 data. ISPRS Journal of Photogrammetry and Remote Sensing, 64, 171-183. Disponível em: <http://dx.doi.org/10.1016/j.isprsjprs.2008.09.00 $4>$.

Redo, D.; Aide, T. M.; Clark, M. L. 2012. Vegetation change in Brazil's dryland ecoregions and the relationship to crop production and environmental factors: Cerrado, Caatinga, and Mato Grosso, 2001-2009. Journal of Land Use Science, 1-31.

Ridwansyah, I. et al. 2020. The impact of land use and climate change on surface runoff and groundwater in Cimanuk watershed, Indonesia. Limnology, n. 0123456789. Disponível em: <https://doi.org/10.1007/s10201-020-00629-9>.

Saito, N. S. et al. 2016. Uso Da Geotecnologia Para Análise Temporal Da Cobertura Florestal. Cerne, 22, 1-18. Disponível em: <http://www.scielo.br/scielo.php?script=sci_artt 
ext\&pid=S0104-

77602016000100011\&lng=pt\&nrm=iso\&tlng=e $\mathrm{n}>$.

Shi, P. et al. 2019. Land-use changes and check dams reducing runoff and sediment yield on the Loess Plateau of China. Science of The Total Environment, 664, 984-994. Disponível em: <https://linkinghub.elsevier.com/retrieve/pii/S00 48969719305625>.

Silva, B. B.; Galvincio, J. D. Montenegro, S. M. G. L.; Machado, C. C. C.; Oliveira, L. M.M.; Moura, M. S. B. 2013. Determinação por sensoriamento remoto da produtividade primária bruta do perímetro irrigado São Gonçalo - PB. Revista Brasileira de Meteorologia [online]. 28, 57-64. https://doi.org/10.1590/S0102-

77862013000100006
Soares, G.A.S., Galvíncio, J.D. 2020. Using lidar to evaluate ater patterns in urban areas basin: Physiographic characterization of the rio beberibe basin, PE| Uso do lidar para avaliar os padrões hídricos de bacias em áreas urbanas: Caracterização fisiográfica da bacia do rio beberibe-PE. Revista Brasileira de Geografia Fisica, 13, 3659-3674.

Wang, Q. et al. 2020. Individual and combined impacts of future land-use and climate conditions on extreme hydrological events in a representative basin of the Yangtze River Delta, China. Atmospheric Research, 236, 163.

Wulder, M. A. et al. 2006. An accuracy assessment framework for large-area land cover classification products derived from medium-resolution satellite data. International Journal of Remote Sensing, 27, 663-683.

Zaidi, S. M. et al. 2017. Landsat-5 time series analysis for land use/land cover change detection using NDVI and semi-supervised classification techniques. Polish Journal of Environmental Studies, 26, 2833-2840. 